\title{
Sur une nouvelle espèce cavernicole du genre Cyathura (Isopoda, Anthuridae), (Résultats zoologiques de la Mission spéléologique britannique en Papouasie-Nouvelle Guinée, 1975, 7).
}

\section{Stoitze Andreev*}

\author{
SUMMARY
}

The present paper deals with the description of a new species of the genus Cyathura - C. beroni n.sp. - discovered in the caves in Papua New Guinea.

$\mathrm{Au}$ cours d'une mission spéléologique britannique dans les différentes régions karstiques de la Papouasie-Nouvelle Guinée le biospéleologiste bulgare Dr. P. Beron a recueilli quelques exemplaires d'Isopodes aquatiques appartenant à une nouvelle espèce du genre Cyathura Norman \& Stebbing, 1886.

D'après les connaissances actuelles, le genre Cyathura est représenté par 14 espèces, dont 4 habitent les eaux douces souterraines.

La première mention du genre Cyathura d'eaux douces est due à Stork (1940). Cet auteur signale la présence d'une nouvelle espèce - Cyathura curassavica - découvert dans les sources d'une région karstique de Curaçao. Beaucoup plus tard Chappuis, Delamare-Deboutteville et Paulian (1956) ont décrit l'espèce C. milloti provenant d'une résurgence du système interstitiel dans l'ile de la Réunion. En 1965 T. Bowman a publié la troisième espèce d'eau douce - C. specus, - provenant d'une grotte de Cuba. Enfin d'après le matériel récolté pendant une mission spéléologique italienne au Mexique Argano (1972) a décrit l'espèce C. shordonii.

Les recherches spéléologiques dans les grottes de la Nouvelle Guinée effectuées par la mission britannique, ont permis de découvrir une faune souterraine riche et remarquable. La nouvelle espèce $C$. beroni recueillie dans les grottes atteignant envi-

*Musée National d'Histoire Naturelle, 1 boul, Rouski, Sofia 1000, Bulgarie. 

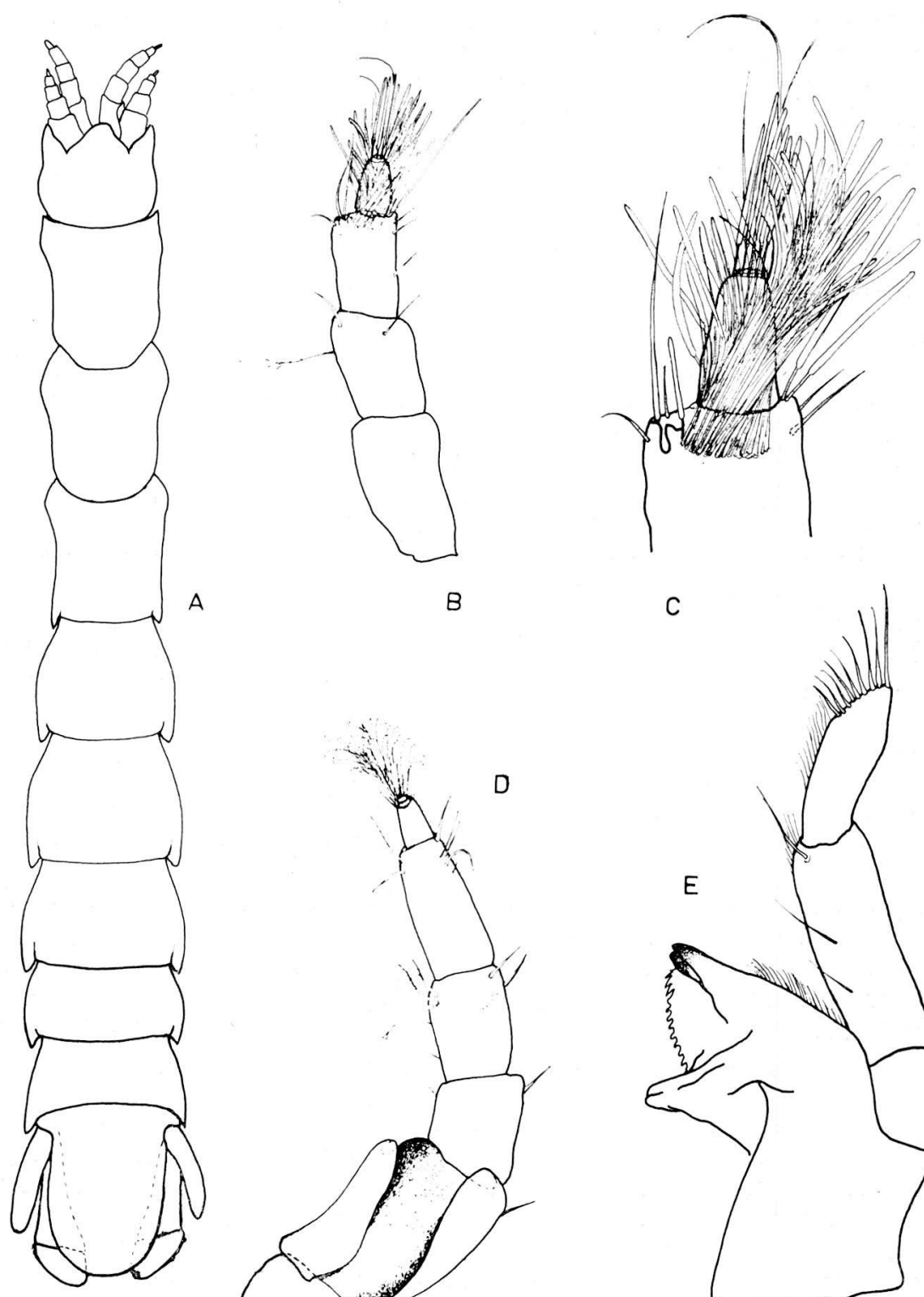

A

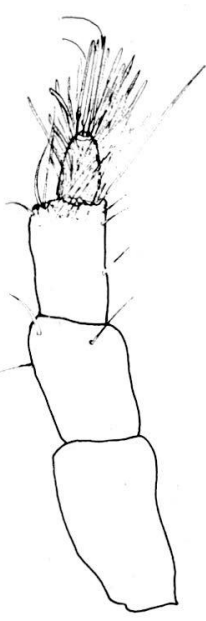

B
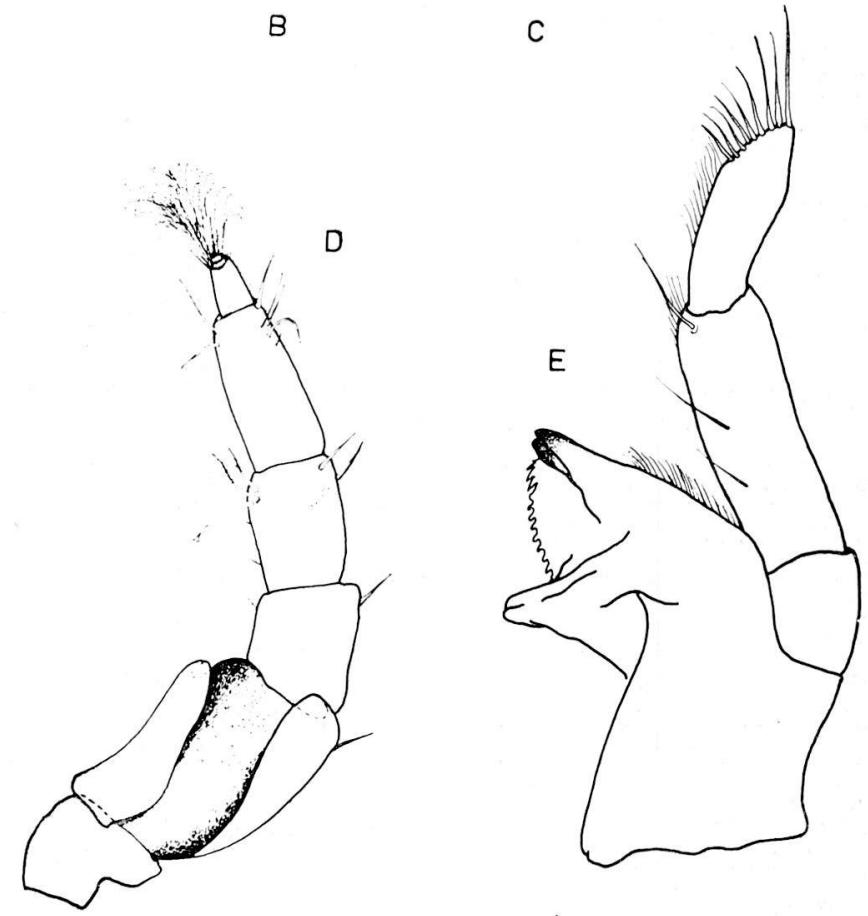

Fig. 1 - Cyathura beroni, n. sp. $0^{-1}$ - A. corps: B. Antenne 1; C. Antenne 1 - dernier segment; D, Antenne 2; E. Mandibule. 
ron $2400 \mathrm{~m}$. d'altitude n'en fait aucune exception. Sa description représente l'objet de la cette note.

Je profite de l'occasion pour exprimer mes remerciements les plus sincères à mon ami et collège M.P. Beron qui a eu la gentillesse de me remettre cette espèce intéressante.

\section{Cyathura beronii n.sp.}

\section{Matériel examiné}

Les Cyathures étudiés sont habitants des rivières souterraines des grottes en Papouasie Nouvelle Guinée. Stations: La grotte Selminum Tem (altitude $2400 \mathrm{~m}$. env.). Western province, 30. VIII.1975, $3 \sigma^{\top}, 1$, leg. P. Beron; 6.IX.1975, $20^{\top}, 1 \%$, leg. P. Beron. La grotte Ogon II, v. Goglme, Chimbru province. 30.X.1975, 10, 3 , , leg. P. Beron. La grotte Warfertem, Emuk Range. On surface of silt in drip pool, Bottom of side pass. 17.II.1978, 1 . . D.Z.

L'holotype et 2 paratypes (mâle et femelle) sont conservés dans les collections du Museum National d'Histoire Naturelle à Sofia. Deux paratypes (mâle et femelle) sont conservés dans le Zoölogish Museum Amsterdam. Les autres paratypes et exemplaires sont déposés dans le British Museum (Natural History).

\section{Description}

Holotype $\sigma^{\top}$, Aveugle. Longeur $8 \mathrm{~mm}$. Corps grêle, allongé, de couleur blanc jaunâtre, sans aucune trace de pigment. Antennes 1 et 2 courtes.

Antenne 1. Le flagellum composé de 3 articles. Le premièr article un peu plus long que la moitié du dernier article du pédoncule et beaucoup plus étroit. Deuxième et troisième articles sont fortement réduits, plus larges que longs. Troisième article garni distalement de 5 longues aesthetasces. Pédoncule comprend 3 articles subégaux et portant dans la zone distale 3-5 soies. (Fig. 1, B, C).

Antenne 2 (Fig. 1, D) plus longue que l'antenne 1. Pedoncule composé de 5 articles recouverts de fins cils. Le deuxième article plus long et plus large que le premièr. Les trois articles suivants sont plus étroits et pourvus de longues soies distalement. Le cinquième est plus long que les autres, armé d'un pinceau de longues soies. Flagellum composé de 4 articles, le premièr article plus long que les suivants, qui sont très courts et larges.

Mandibule (Fig. 1, E) - sans lame mobile; pars incisiva avec deux dents; bord tranchant armé de 16 dents courtes et recourbées. Derrière le bord tranchant se trouve un lobe bien développé. Deuxième article du palpe plus long que le premier et le troisième. Le dernièr article a son borde interne portant une 


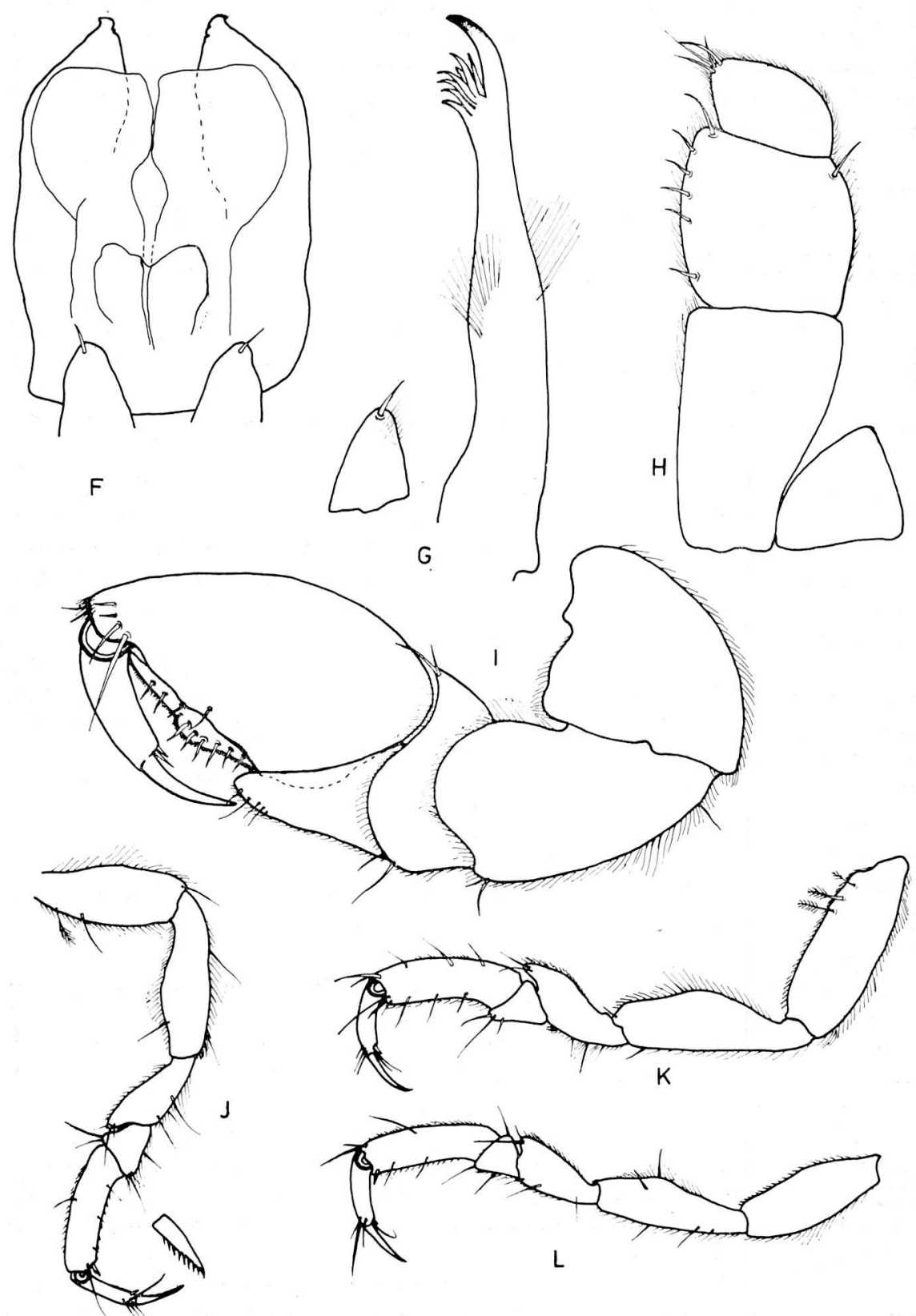

Fig. 2 - Cyathura beroni n. sp. $0^{\top}$ F, Maxille 2; G, Maxille 1; H. Maxillipède; I. Péréiopodes 1; J - L, Péréiopodes III, I et V. 
rangée de soies fines et 10 longues soies dans la région apicale.

Maxille 1: bilobée. Lobe externe bien développé dont la partie distale possède 7 épines, dont la dernière est la plus forte; la partie médiale garnie de fins poils. Lobe interne petit, oviforme avec une courte épine. (Fig. 2, G).

Maxille 2 (Fig. 2, F) et hypopharynx coalescents; la partie apicale pourvue d'une courte épine recourbée en dehors.

Maxillipède (Fig. 2, H) représenté par un lobe interne bien développé et un exopodite en forme triangulaire. Exopodite ne dépasse pas la moitié du premier article de l'endopodite. Premier article de l'endopodite à peu près 2 fois plus long que large. Deuxième article plus court que le premier et portant à son bord interne 5-6 épines à l'angle distal. Troisième article, relativement court et montrant quelques soies et beaucoup de poils apicalement. Les deux derniers articles sont recouverts de fins cils.

Le premier et le deuxième péréionites subégaux. Bords marginaux des péréionites recouverts de cils et de 2-3 longues soies.

Péréiopode 1 (Fig. 2, I) robuste. Propodite piriforme, bien développé. Bord palmaire ayant dans sa partie mèdiale un lobe bien sailliant, armé de 9-10 épines et courts cils. Carpe trianguiaire avec quelques soies subdistales.

Péréiopodes 2-7 (Fig. 2, J, K, L) plus longs et allongés. Bords marginaux avec des articles pubescents. Les propodites à leurs bords internes ainsi que distalement portent une épine carriée. Péreiopode 2 est le plus long. Les autres diminuent progressivement.

Les 5 premiers segments du pléon complètement coalescents ainsi que le sixième segment et le telson.

Pléopode 1 (Fig. 3, M) - Exopodite ovoide; bord interne droit et armé de fins poils; bord externe oval pourvu de 15-18 soies pennées.

Pléopode 2 trilobé. Exopodite (Fig. 3, N) trois fois plus long que large, avec 5-7 épines distalement. L'endopodite transparent, plus court que l'exopodite. Apophyse longue et grêle à peu près égale à l'exopodite. La partie apicale faiblement élargie et bifurquée (Fig. 3, O, R).

Uropode (Fig. 3, P) - Exopodite plus court que la moitié du telson. Son bord interne avec une rangée de soies dans la partie distale, le bord externe garni de fines soies et de 2-3 épines. Endopodite plus grand et dépassant la longeur du telson, pourvu de très longues soies.

Telson (Fig. 3, Q) linguiforme avec des bords parallèles. La région apicale porte 2-3 longues soies et quelques plus courtes. A proximité de la base du telson se trouvent deux statocystes bien saillants. 


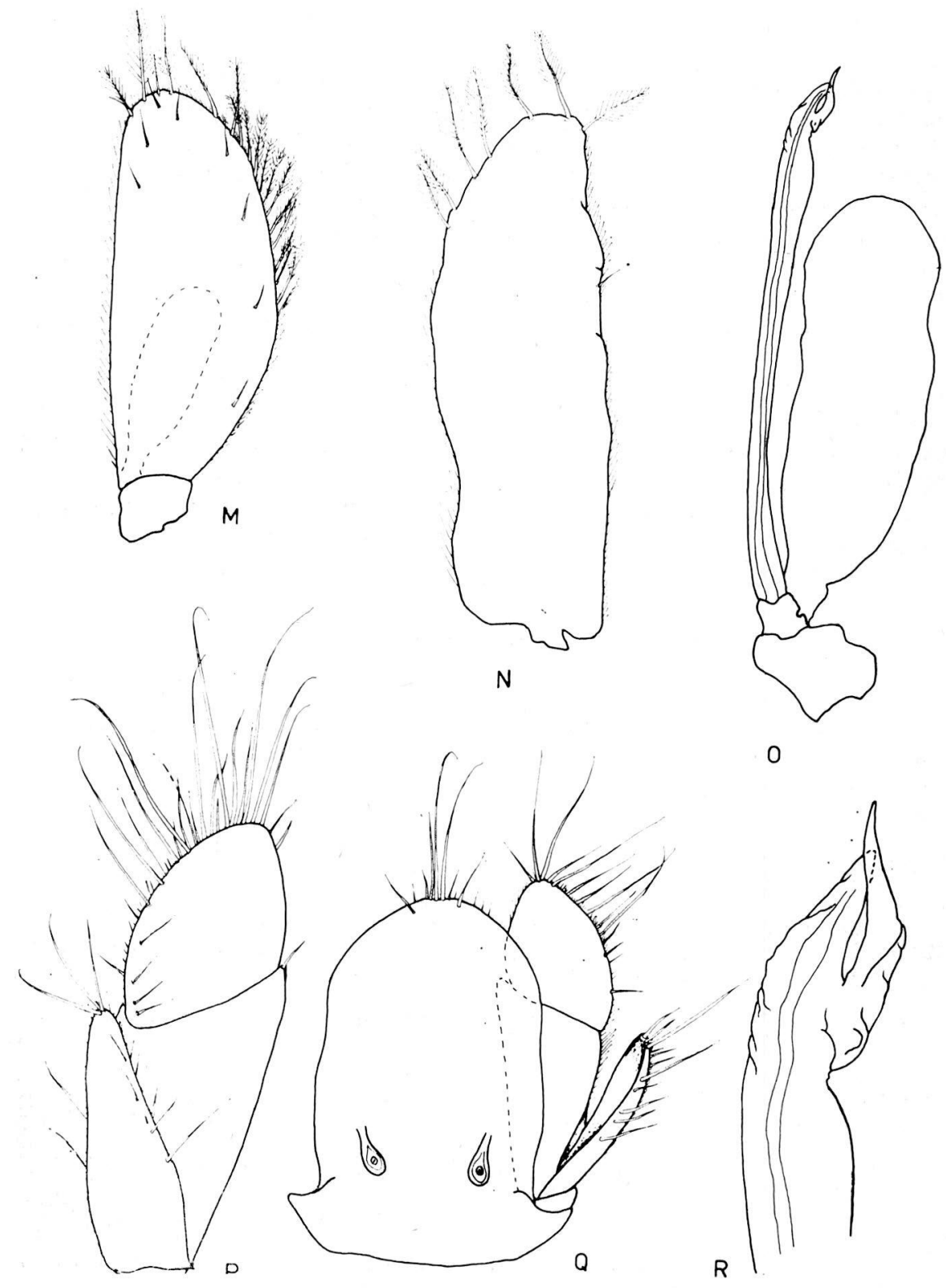

Fig. 3 - Cyathura beroni n. sp. $\sigma^{\star}$ - M. pléopode 1; N. Pléopode 2 exopodite; O. Pléopode 2 endopodite et apophyse; P. Uropode; Q. Telson; R. Pléopode 2, apophyse, partie apicale à un grossissement plus fort. 


\section{Affinités}

Partant des connaissances actuelles sur le genre Cyathura Argano (1972) propose une division relative des espèces en 3 groupes fondamentaux se basant sur certains caractères morphologiques. Les deux premiers groupes comprennent les espèces marines tandis que le troisième est composé des espèces habitant les eaux douces souterraines et cavernicoles. En dehors de cette subdivision l'auteur assigne une place à part à C. millot.

C. beroni n.sp. selon certains caractères, approche les espèces du troisième groupe: C. curassavica, C. specus et C. sbordonii, comme par example le sixième segment du pléon et le telson coalescents, l'aspect géneral et la chétotaxie des pièces buccales et enfin la structure commune de certains appendices. Néamoins la nouvelle espèce diffère distinctement des espèces, citées ci-dessus par les caractères suivants: - lobe bien développé dans la partie médiale du bord palmaire du péréiopode 1 , bords parallèles du telson et surtout la structure du pléopode 2.

\section{RESUME}

L'objet de la présente note est la description d'une nouvelle espèce du genre Cyathura - C. beroni n.sp. - découverte dans les grottes de la Papouasie-Nouvelle Guinée.

\section{BIBLIOGRAPHIE}

ARGANO, R. 1971. Cythura sbordonii, nuova specie cavernicola del Messico sudorientale. Diagnosi preliminare (Crustacea, Isopoda, Anthuridae), Fragmenta Entomologica, 7: 303-305.

ARGANO, R. 1972. On a troglobitic Cyathura from subterranean waters of Mexico (Crustacea, Isopoda). Problemi attuali di scienza e di cultura, Quaderno 171: 23-34.

BARNARD, K. 1925. A Revision of Family Anthuridae (Crustacea, Isopoda), with Remarks of certain Morphological Peculiarities. J. Linn. Soc. London, 36: $109-160$.

BOWMAN, T. 1965. Cyathura spécus, a new cave isopode from Cuba (Anthuroidea: Anthuridae). Studies on the fauna of Curaçao and other Caribbean islands 22: 88-97.

CHAPPUIS, P., Cl. DELAMARE-DEBOUTTEVILLE et R. PAULIAN, 1956. Crustacés des eaux souterraines littorales d'une résurgence d'eau douce à la Réunion. Mémoires de l'Institut scientifique du Madagascar, (A) 11: 51-78.

CLERET, J. 1959. Polytypisme antennulaire et rapport numérique des sexes chez Cyathura carinata (Kröyer) (Isopoda, Anthuridae). C.R. Acad. Sc. 248: 2508-2510.

MENZIES, R. 1951. New marine Isopods, chiefly from Northern California, with notes on related forms. Proc. U.S. Nat. Mus., 101, 3273: 105-156.

STORK, A. 1940. A new fresh-water isopod from Curaçao. Studies on the fauna of Curaçao, Aruba, Bonaire and the Venezuelan Islands, 2: 147-150. 